\title{
Polar OwnIndex is not a reliable indicator of aerobic training status
}

\author{
F Köteles ${ }^{1}$, Z Dömötör ${ }^{2}$, T Berkes ${ }^{1}$, R Szemerszky $^{1}$ \\ ${ }^{1}$ Institute of Health Promotion and Sport Sciences, Eötvös Loránd University, Budapest, Hungary \\ ${ }^{2}$ Doctoral School of Psychology, Eötvös Loránd University, Budapest, Hungary
}

Received: July 26, 2015

Accepted: September 18, 2015

\begin{abstract}
Polar watches with heart rate monitoring function have become popular among recreational and professional athletes. In addition to monitoring functions, they calculate a specific index called OwnIndex which is claimed to measure aerobic training status. The current research attempted to shed light on the factors determining the OwnIndex. In Study 1, OwnIndex calculated by the RS-400 Polar watch was estimated using anthropometric (gender, age, height, weight), cardiovascular (resting HR, RMSSD), and exercise-related (maximal oxygen uptake, self-reported physical activity) data of 45 young adults. In Study 2, the OwnIndex was measured in 21 young adults twice, first with self-reported physical activity set to the lowest, then to the highest value. In the regression analysis (Study 1), the only significant predictor of OwnIndex was self-reported physical activity $\left(\mathrm{R}^{2}=0.883 ; \beta=0.915, p<\right.$ $0.001)$. A significant difference with a large effect size $(t(20)=-16.657, p<0.001, d=3.635)$ and no significant correlation $(r=-0.32 ; p=0.155)$ were found between the OwnIndices calculated with different levels of activity in Study 2. As anthropometric and cardiac variables play a practically negligible role in the calculation of the OwnIndex, it cannot be considered an appropriate measure of aerobic fitness.
\end{abstract}

Keywords: aerobic fitness, assessment, technology, exercise, placebo

Monitoring of heart rate (HR) has become more and more popular among recreational as well as elite athletes in aerobic sports, particularly in cycling and running. Relying on this information, athletes are able to optimize their training load, and avoid cardiac overload. Although even modern mobile phones are able to receive and handle HR-related information, the primary platform for such tasks is a combination of a chest strap that functions as electrodes, a wireless transmitter attached to the strap, and a receiver unit, usually a special watch. The development of these devices started already in the 70s, and today several companies offer products that support the planning and monitoring of workouts. One of the leading companies is Polar Electro; its advanced watches are claimed not only to be able to monitor heart rate during exercise but also to characterize aerobic fitness (i.e., maximal oxygen uptake: $\mathrm{VO}_{2} \max$ ) by a specific index called OwnIndex. The OwnIndex is calculated using a non-linear method, i.e., an artificial neural network (ANN) approximation from demographic and heart rate related variables. This latter component is not specified in more detail, i.e., the actual variables (e.g. heart rate, certain types of heart rate variability, HRV) used in the approximation are unknown. According to the reported results, the method was able to estimate the $\mathrm{VO}_{2}$ max from a short R-R interval measurement with high correlation (0.96) and accuracy (39-41). In the current implementation of the method, the calculation of

Corresponding author: Dr. habil. Ferenc Köteles, $\mathrm{PhD}$, associate professor

Institute of Health Promotion and Sport Sciences, Eötvös Loránd University, Budapest, Hungary

Bogdánfy Ö. u. 10, H-1117 Budapest, Hungary

Phone: +36-1-2090619; E-mail: koteles.ferenc@ppk.elte.hu 
OwnIndex is based on user-specific data (gender, age, body weight and height, maximal oxygen uptake (if available), minimum (resting) and maximum HR, and average physical activity in the last 6 months) and an approximately 5 minutes long HR measurement (called Polar Fitness Test) in resting state and position (preferably in the morning, under relaxed conditions). Polar Electro claimed that the Polar OwnIndex is "as reliable as any other submaximal fitness test" (30).

According to our current knowledge, a possible (however, not always reliable) cardiac indicator of aerobic training status is resting heart rate (rHR): regular endurance exercise training results in a slight or marked decrease in rHR, depending on the training load $(6,9$, $10,18,35,36)$. Measures of vagal influence on heart rate variability (HRV; in frequency domain: high frequency component of the total variability, HF-HRV, in time domain: root mean square of the successive differences, RMSSD) as possible markers of training status and aerobic fitness have also received special attention. Although promising positive findings were reported $(2,22,24)$, the results are not yet conclusive for many reasons. First, vagalrelated heart rate variability was found to be higher in moderately trained subjects than in sedentary individuals; however, elite athletes showed no significantly better values than the latter group (9), or were characterized by individual temporal patterns (29). Second, endurance training had no impact on vagal HRV indices in several studies, which might be explained by methodological issues (e.g., duration of recordings, body positioning during recording, characteristics of training load) (6). Third, daily recordings averaged over weeks proved to be better indicators of training status than single measurements that show considerable day-today variability $(23,29)$. In conclusion, measures of heart rate in themselves (and particularly if they are calculated form a single measurement) do not seem to be appropriate indicators of training status in aerobic sports $(8,23,28)$.

According to the reported findings (which typically used linear statistical methods), the gap between the accuracy of linear and non-linear approximations of $\mathrm{VO}_{2}$ max from HRrelated variables appears to be surprisingly large. In the current study, the accuracy of the OwnIndex as an indicator of $\mathrm{VO}_{2}$ max was investigated. The research comprised two studies designed to investigate the linear contribution of anthropometric, and heart- and exerciserelated variables to the Polar OwnIndex. More specifically, it was hypothesized that gender, age, body weight and height, maximal oxygen uptake, resting HR, RMSSD, and average physical activity in the last 6 months would be connected to the Polar OwnIndex, and able to reliably predict it.

\section{Materials and Methods}

\section{Participants}

The sample of Study 1 consisted of 45 university students (17 males; mean age: $21.24 \pm 1.60$ yrs; 19-26 yrs) from Eötvös Loránd University, Budapest, Hungary. Their participation was a part of a seminar in exercise physiology. The sample of Study 2 represented a randomly chosen subgroup of the former sample $(N=21,12$ males, mean age $=20.43 \pm 0.99$ yrs; $19-23$ yrs). Participants received detailed information before the study, and signed an informed consent form. The study was approved by the institutional ethical board of the faculty.

\section{Measurements}

Maximal oxygen uptake $\left(\mathrm{VO}_{2} \max \right)$ as an indicator of aerobic training status was measured using a Daum Ergo Bike Premium 8i bicycle ergometer (manufactured by Daum Electronic 
GmbH, Fürth, Germany) connected to a Fitmate PRO ergospirometer device (Cosmed Srl, Rome, Italy). The built-in automatic graded exercise test with a continuous $85 \%$ submaximal protocol was used. A three-minute warm-up of pedaling with $25 \mathrm{~W}$ workload was followed by the exercise phase with escalating workload with a duration of 12 minutes. Maximal HR was calculated using the formula of 220 - age. During the escalating phase, pedaling rate was kept at approximately $75 \mathrm{rev} *$ min-1. Gas exchange and ventilator variables were measured by the ergospirometer. These data were recorded every thirty seconds. Heart rate was monitored throughout the test by a HR belt which sent the HR signals to a HR receiver connected to the Fitmate device. The gas sensor of the ergospirometer was calibrated before each test. Tests were stopped by the ergospirometer.

Electrocardiogram (ECG) was recorded using a Nexus-4 device (Mind Media BV, Herten, the Netherlands). ECG data was obtained with a sampling rate of $1024 \mathrm{~Hz}$ using the modified lead II electrode placement (distal end of the right collarbone and lower left rib). The rHR and RMSSD indices were calculated using the KubiosHRV software v2.2 (5).

OwnIndex was calculated using the Polar Fitness Test of RS-400 Polar watch and the matching chest strap and transmitter (POLAR WearLink; Polar Electro, Kempele, Finland).

Body weight and height were measured by the experimenters before Study 1 .

Physical activity level was determined following the instructions of the Polar Fitness Test on a 4-point scale: low (no regular aerobic training), moderate (0.5-2 hours of aerobic training per week, e.g., 5-10 km running), high (2-4 hours of aerobic training/10-40 km running per week), and top (heavy physical exercise at least 5 times a week).

\section{Procedure}

In Study 1, participants were measured one by one in a separate room. In the first measurement, body weight and height were measured, and then participants completed the $85 \%$ submaximal oxygen uptake test. In the second measurement (7-14 days later), participants set their personal data (including the measured $\mathrm{VO}_{2}$ max value) in the Polar watch, then the Polar chest strap with the transmitter and the three electrodes for the ECG measurement were placed on their body. ECG data was recorded simultaneously with the Polar Fitness Test protocol in a lying body position under resting conditions.

In Study 2, participants were asked to enter their personal data (age, gender, height, and weight) in the RS-400 Polar watch. $\mathrm{VO}_{2} \max$, resting and maximum heart rate were left untouched (i.e., the default values of the device were used), activity level was set to the lowest value ("1"), and the OwnIndex test was conducted in a lying position under relaxed conditions (condition 1). After finishing the test, participants set activity level to the highest value ("4"), all other data remained unchanged, and the test was completed again (condition 2).

\section{Statistical analysis}

Statistical analysis was carried out using the SPSS v21 software. As variables showed no deviation from normal distribution in either study, parametric methods were used in the statistical analysis. In Study 1, Pearson correlation was used to estimate relationships among continuous variables, and Spearman correlation for binary and ordinal variables. To investigate variables' independent contributions to OwnIndex, a multiple linear regression analysis was conducted. Variables were entered into the equation in two steps (the ENTER method was used): (Step 1) gender ( 0 = male, 1 = female), age, height, and weight; (Step 2) $\mathrm{rHR}, \mathrm{RMSSD}, \mathrm{VO}_{2}$ max, and activity. Another regression analysis was run to predict $\mathrm{VO}_{2} \max$. 
In this analysis, gender, age, height, and weight were entered in Step 1, and rHR, RMSSD, OwnIndex, and activity were entered in Step 2. In Study 2, the two sets of OwnIndices were compared in two ways: using a paired-samples $t$-test (group level change) and Pearson correlation (individual level connection).

\section{Results}

Descriptive statistics of Study 1 were presented in Table I; results of the correlation analysis are summarized in Table II. The OwnIndex showed significant correlations with $\mathrm{VO}_{2} \mathrm{max}$ $(r=0.33, p=0.027)$ and physical activity $(\rho=0.91, p<0.001)$, however, it was not connected to age, gender, body weight and height, $\mathrm{rHR}$, and RMSSD. $\mathrm{VO}_{2}$ max correlated with gender ( $\rho=-0.34, p=0.021)$, body height $(r=0.49, p=0.001)$, and reported physical activity $(r=0.33, p=0.025)$, but not with rHR and RMSSD.

Table I. Descriptive statistics of the measured variables

\begin{tabular}{|l|c|c|}
\hline & Mean & Std. Deviation \\
\hline Body height (whole sample) & 1.73 & 0.128 \\
\hline Body height (males) & 1.84 & 0.087 \\
\hline Body height (females) & 1.66 & 0.098 \\
\hline Body weight (whole sample) & 65.40 & 11.244 \\
\hline Body weight (males) & 77.23 & 6.584 \\
\hline Body weight (females) & 58.22 & 6.228 \\
\hline VO $_{2}$ max (whole sample) & 40.90 & 10.128 \\
\hline VO $_{2}$ max (males) & 46.17 & 12.957 \\
\hline VO ${ }_{2}$ max (females) & 37.70 & 6.290 \\
\hline Resting HR (1/min) & 62.69 & 8.345 \\
\hline RMSSD (ms) & 73.81 & 35.517 \\
\hline Physical activity & 2.84 & 0.706 \\
\hline OwnIndex & 50.18 & 8.77 \\
\hline
\end{tabular}

Table II. Results of the correlation analysis. Spearman coefficients $(\rho)$ for gender and physical activity, and Pearson coefficients $(r)$ for all other variables

\begin{tabular}{|l|c|c|}
\hline & OwnIndex & VO $_{2} \mathbf{m a x}$ \\
\hline Age & $r=0.13, p=0.401$ & $r=0.24, p=0.111$ \\
\hline Gender & $\rho=0.15, p=0.336$ & $\rho=-0.34, p=0.021$ \\
\hline Body weight & $r=-0.06, p=0.686$ & $r=0.29, p=0.054$ \\
\hline Body height & $r=-0.12, p=0.450$ & $r=0.49, p=0.001$ \\
\hline rHR & $r=0.14, p=0.361$ & $r=-0.06, p=0.673$ \\
\hline RMSSD & $r=-0.10, p=0.520$ & $r=-0.16, p=0.306$ \\
\hline Physical activity & $\rho=0.91, p<0.001$ & $r=0.33, p=0.025$ \\
\hline
\end{tabular}


The first equation of the regression analysis with OwnIndex as outcome variable was not significant. The second equation explained $88.3 \%$ of the total variance $(p<0.001)$, the only significant predictor of OwnIndex was physical activity $(\beta=0.915, p<0.001)$ (see Table III for details).

The first equation of the second regression analysis explained $34.4 \%$ of the total variance of $\mathrm{VO}_{2} \mathrm{max}$ with the significant contribution of body height $(\beta=0.504, p=0.009)$. The second equation explained $49.1 \%(p=0.001)$, however, the only significant contributor remained body height $(\beta=0.550, p=0.004)$. Contribution of OwnIndex was not even close to a significant level $(\beta=0.370, p=0.287)$ (Table IV).

Table III. Results of the multiple linear regression analysis with OwnIndex as dependent variable

\begin{tabular}{|c|c|c|c|c|}
\hline & $\begin{array}{c}\text { Step 1 } \\
\mathbf{R}^{2}=\mathbf{0 . 0 4 2}, p=\mathbf{0 . 7 8 2}\end{array}$ & & $\begin{array}{c}\text { Step 2 } \\
\Delta \mathbf{R}^{2}=\mathbf{0 . 8 4 1 ,} p<\mathbf{0 . 0 0 1}\end{array}$ & \\
\hline & $\mathrm{B} \pm \mathrm{SE}$ & $\beta$ & $\mathbf{B} \pm \mathbf{S E}$ & $\beta$ \\
\hline Gender & $3.994 \pm 5.313$ & .223 & $1.265 \pm 2.070$ & .071 \\
\hline Age & $.792 \pm .870$ & .144 & $-.386 \pm .354$ & -.070 \\
\hline Height & $-4.925 \pm 15.162$ & -.072 & $1.464 \pm 6.519$ & .021 \\
\hline Weight & $.140 \pm .225$ & .179 & $-.016 \pm .088$ & -.020 \\
\hline rHR & & & $.001 \pm .068$ & .001 \\
\hline RMSSD & & & $-.006 \pm .016$ & -.025 \\
\hline Activity & & & $11.367 \pm .826$ & $.915 * * *$ \\
\hline $\mathrm{VO}_{2} \max$ & & & $.074 \pm .068$ & .085 \\
\hline
\end{tabular}

$* * * p<0.001$

Table IV. Results of the multiple linear regression analysis with maximal oxygen uptake $\left(\mathrm{VO}_{2} \max \right)$ as dependent variable

\begin{tabular}{|c|c|c|c|c|}
\hline & $\begin{array}{c}\text { Step 1 } \\
\mathbf{R}^{2}=\mathbf{0 . 3 4 4 ,} p=\mathbf{0 . 0 0 2}\end{array}$ & & $\begin{array}{c}\text { Step 2 } \\
\Delta \mathbf{R}^{2}=\mathbf{0 . 1 4 8 ,} p=\mathbf{0 . 0 5 2}\end{array}$ & \\
\hline & $\mathrm{B} \pm \mathrm{SE}$ & $\beta$ & $\mathrm{B} \pm \mathrm{SE}$ & $\beta$ \\
\hline Gender & $-5.324 \pm 5.080$ & -.258 & $-6.849 \pm 4.881$ & -.332 \\
\hline Age & $1.597 \pm .832$ & .252 & $1.311 \pm .839$ & .207 \\
\hline Height & $39.943 \pm 14.496$ & $.504 * *$ & $43.550 \pm 13.941$ & $.550 * *$ \\
\hline Weight & $-.225 \pm .215$ & -.249 & $-.267 \pm .206$ & -.297 \\
\hline $\mathrm{rHR}$ & & & $-.007 \pm .163$ & -.006 \\
\hline RMSSD & & & $.020 \pm .038$ & .072 \\
\hline Activity & & & $.381 \pm 4.979$ & .027 \\
\hline OwnIndex & & & $.427 \pm .395$ & .370 \\
\hline
\end{tabular}

$* * p<0.01$

In Study 2, $t$-test showed a significant difference between the two measurements with a large effect size (low activity: $\mathrm{M}=32.00, \mathrm{SD}=4.483$; high activity: $\mathrm{M}=59.38, \mathrm{SD}=4.780$; $t(20)=-16.657, p<0.001, d=3.635)$. The Pearson correlation between the two data sets was non-significant $(r=-0.32 ; p=0.155)$. 


\section{Discussion}

According to the results of Study 1, the OwnIndex showed only a medium level correlation with maximal oxygen uptake (explaining approximately $11 \%$ of its variance), which disappeared after controlling for anthropometric and cardiac characteristics, and physical activity. Moreover, the OwnIndex was almost completely determined by the reported level of physical activity; more than $83 \%$ of its variance was explained by physical activity level in the correlation and regression analyses (after controlling for all other variables).

Study 2 showed that the OwnIndex could easily be manipulated to a great extent by the reported level of physical activity, even in the complete absence of other (i.e., cardiac or anthropometric) changes. In the correlation analysis, the two data sets obtained from the same individuals showed no connection after the change of physical activity. In the group level comparison, change in physical activity substantially changed the OwnIndices; in fact, the calculated OwnIndex was usually evaluated by the software of the watch as low or very low in condition 1, and typically belonged to the very good or elite category in condition 2 for the same person.

The results of the reported two studies do not support the claim that the Polar OwnIndex is an appropriate indicator of aerobic fitness and "as reliable as any other submaximal fitness test" (30). First, the connection between OwnIndex and $\mathrm{VO}_{2} \max$ was weak. Second, even with the use of linear methods, a great proportion of the variability of OwnIndex was explainable only from reported physical activity. Third, anthropometric and cardiac variables had almost no impact on the OwnIndex; the non-existing correlation between the OwnIndices calculated from two almost identical data sets (the only difference between the two sets was the level of reported physical activity) clearly shows that these variables play an inferior role (if any) in the determination of the OwnIndex.

Polar watches have been accepted as appropriate tools to monitor HR in medicine $(1,20$, $27)$ and also in exercise physiology $(4,16,21,38)$. Recently, their utilization in HRV-based studies (typically, in heart diagnostics) has also been reported $(12,13,19,33)$. According to the results of comparison studies, top-level Polar watches (S810, S810i) can generally be considered as valid as traditional ECG in obtaining data for HR and HRV analysis $(14,15$, $25,26,31,32,42,45)$, although limitations were also reported $(25,45)$. In one study, however, the RS-800 system was found to be inferior compared to the traditional method (44).

According to the present results, these generally positive findings are not generalizable to the OwnIndex, at least not in the case of a medium-level device (Polar RS-400). Limitations of the HR assessment (e.g., a low sampling rate, unsatisfactory identification and removal of non-normal heartbeats) can lead to a considerable decrease of reliability of the measurement (37). These procedures require a considerable calculating capacity, which might not be available in a medium-level Polar device. Even if the formula of calculation takes into consideration anthropometric and cardiac variables, these variables play a practically negligible role in the determination of the OwnIndex compared to the reported level of physical activity. Therefore, in spite of its name and claimed meaning, the OwnIndex does not really encompass, measure, or represent an individual characteristic beyond self-reported physical activity.

Unfortunately, measurement of physical activity seems to be inappropriate, as it is based on self-reported (subjective) average of physical activity over a long period (6 months) which might be heavily biased. Second, only duration of regular physical activity but not its type (e.g., aerobic or anaerobic) is rated, which leads to further inaccuracies. Third, a scale with higher resolution (i.e., a 10-point scale instead of the current 4-point scale) would be desirable 
in the case of such an important predictor. As physical activity substantially contributes to the calculation of the OwnIndex, these inaccuracies may cause changes (more precisely: biases and artefacts) in OwnIndex, that might easily be misinterpreted by the users as changes in their aerobic fitness.

According to recent advertisements, top performance in sports does not seem to be achievable without the use of appropriate aids (e.g., magnetic bracelets, kinesiotapes) and monitoring devices. In placebo-controlled studies, these aids and devices usually show no better effectiveness than their respective placebos, and their specific effects are not supported by empirical results $(7,11,17,34,43)$. This is not to say, however, that placebo interventions are ineffective; it is well known that the placebo effect can play a role in the improvement of sport performance (3). From this point of view, these aids and devices are able to mobilize psychological resources which will result in better performance. In the light of the present results, the Polar OwnIndex can be considered as a "technical placebo": more training leads to the increase of the OwnIndex, this offers a direct and positive feedback for the athlete who tries to train more and more for better OwnIndex values.

\section{Acknowledgement}

This research was supported by the Hungarian National Scientific Research Fund (OTKA K 109549).

\section{REFERENCES}

1. Arazpour M, Bani MA, Hutchins SW, Jones RK: The physiological cost index of walking with mechanical and powered gait orthosis in patients with spinal cord injury. Spinal Cord 51, 356-359 (2013)

2. Baumert M, Brechtel L, Lock J, Voss A: Changes in heart rate variability of athletes during a training camp. Biomed. Tech. (Berl) 51, 201-204 (2006)

3. Bérdi M, Köteles F, Szabó A, Bárdos G: Placebo effects in sport and exercise: a meta-analysis. European Journal of Mental Health 6, 196-212 (2011)

4. Berg K, Narazaki K, Latin R, Vincent W, Meisinger M, Sjoberg C, Kaufman C: Oxygen cost and energy expenditure of racquetball. J. Sports Med. Phys. Fitness 47, 395-400 (2007)

5. Biosignal Analysis and Medical Imaging Kubios HRV. http://kubios.uef.fi/. (2014)

6. Borresen J, Lambert MI: Autonomic control of heart rate during and after exercise: measurements and implications for monitoring training status. Sports Med. 38, 633-646 (2008)

7. Broatch JR, Petersen A, Bishop DJ: Postexercise cold-water immersion benefits are not greater than the placebo effect. Med. Sci. Sports Exerc. 46, 2139-2147 (2014)

8. Buchheit M: Monitoring training status with HR measures: do all roads lead to Rome? Front. Physiol. 5, 73 (2014)

9. Buchheit M, Simon C, Piquard F, Ehrhart J, Brandenberger G: Effects of increased training load on vagalrelated indexes of heart rate variability: a novel sleep approach. Am. J. Physiol. Heart Circ. Physiol. 287, H2813-H2818 (2004)

10. Carter JB, Banister EW, Blaber AP: Effect of endurance exercise on autonomic control of heart rate. Sports Med. 33, 33-46 (2003)

11. Chang HY, Cheng SC, Lin CC, Chou KY, Gan SM, Wang CH: The effectiveness of kinesio taping for athletes with medial elbow epicondylar tendinopathy. Int. J. Sports Med. 34, 1003-1006 (2013)

12. Corrêa PR, Catai AM, Takakura IT, Machado MN, Godoy MF: Heart rate variability and pulmonary infections after myocardial revascularization. Arq. Bras. Cardiol. 95, 448-456 (2010)

13. Curilem Gatica C, Almagià Flores A, Yuing Farías T, Rodríguez Rodríguez F: Body composition and heart rate variability in patients with chronic obstructive pulmonary disease pulmonary rehabilitation candidates. Nutr. Hosp. 30, 179-182 (2014)

14. Gamelin FX, Baquet G, Berthoin S, Bosquet L: Validity of the polar S810 to measure R-R intervals in children. Int. J. Sports Med. 29, 134-138 (2008) 
15. Gamelin FX, Berthoin S, Bosquet L: Validity of the polar S810 heart rate monitor to measure R-R intervals at rest. Med. Sci. Sports Exerc. 38, 887-893 (2006)

16. Ham SA, Reis JP, Strath SJ, Dubose KD, Ainsworth BE: Discrepancies between methods of identifying objectively determined physical activity. Med. Sci. Sports Exerc. 39, 52-58 (2007)

17. Heneghan C, Howick J, O’Neill B, Gill PJ, Lasserson DS, Cohen D, Davis R, Ward A, Smith A, Jones G, Thompson M: The evidence underpinning sports performance products: a systematic assessment. BMJ 2, e001702 (2012)

18. Huang G, Shi X, Davis-Brezette JA, Osness WH: Resting heart rate changes after endurance training in older adults: a meta-analysis. Med. Sci. Sports Exerc. 37, 1381-1386 (2005)

19. Kunz VC, Souza RB, Takahashi AC, Catai AM, Silva E: The relationship between cardiac autonomic function and clinical and angiographic characteristics in patients with coronary artery disease. Rev. Bras. Fisioter 15, 503-510 (2011)

20. Leung AKL, Wong AFY, Wong ECW, Hutchins SW: The Physiological Cost Index of walking with an isocentric reciprocating gait orthosis among patients with $\mathrm{T}(12)-\mathrm{L}(1)$ spinal cord injury. Prosthet. Orthot. Int. 33, 61-68 (2009)

21. Loftin M, Anderson P, Lytton L, Pittman P, Warren B: Heart rate response during handball singles match-play and selected physical fitness components of experienced male handball players. J. Sports Med. Phys. Fitness 36, 95-99 (1996)

22. De Meersman RE: Heart rate variability and aerobic fitness. Am. Heart J. 125, 726-731 (1993)

23. Le Meur Y, Pichon A, Schaal K, Schmitt L, Louis J, Gueneron J, Vidal PP, Hausswirth C: Evidence of parasympathetic hyperactivity in functionally overreached athletes. Med. Sci. Sports Exerc. 45, 2061-2071 (2013)

24. Nummela A, Hynynen E, Kaikkonen P, Rusko H: Endurance performance and nocturnal HRV indices. Int. J. Sports Med. 31, 154-159 (2010)

25. Nunan D, Donovan G, Jakovljevic DG, Hodges LD, Sandercock GR, Brodie DA: Validity and reliability of short-term heart-rate variability from the Polar S810. Med. Sci. Sports Exerc. 41, 243-250 (2009)

26. Nunan D, Jakovljevic DG, Donovan G, Hodges LD, Sandercock GR, Brodie DA: Levels of agreement for RR intervals and short-term heart rate variability obtained from the Polar S810 and an alternative system. Eur. J. Appl. Physiol. 103, 529-537 (2008)

27. Oliveira NL, Ribeiro F, Teixeira M, Campos L, Alves AJ, Silva G, Oliveira J: Effect of 8-week exercise-based cardiac rehabilitation on cardiac autonomic function: A randomized controlled trial in myocardial infarction patients. Am. Heart J. 167, 753-761.e3 (2014)

28. Plews DJ, Laursen PB, Kilding AE, Buchheit M: Evaluating training adaptation with heart-rate measures: a methodological comparison. Int. J. Sports Physiol. Perform 8, 688-691 (2013)

29. Plews DJ, Laursen PB, Stanley J, Kilding AE, Buchheit M: Training adaptation and heart rate variability in elite endurance athletes: opening the door to effective monitoring. Sports Med. 43, 773-781 (2013)

30. Polar Electro Oy: Polar Fitness Test and OwnIndex http://www.polar.com/en/support/polar_fitness test_and ownindex. Accessed 11 Dec 2014

31. Radespiel-Tröger M, Rauh R, Mahlke C, Gottschalk T, Mück-Weymann M: Agreement of two different methods for measurement of heart rate variability. Clin. Auton. Res. 13, 99-102 (2003)

32. Rezende Barbosa MP, Silva NT, Azevedo FM, Pastre CM, Vanderlei LC: Comparison of Polar( $\left({ }^{\circledR}\right)$ RS $800 G 3\left({ }^{\mathrm{TM}}\right)$ heart rate monitor with Polar( $\left({ }^{R}\right) \mathrm{S} 810 \mathrm{i}\left({ }^{\mathrm{TM}}\right)$ and electrocardiogram to obtain the series of RR intervals and analysis of heart rate variability at rest. Clin. Physiol. Funct. Imaging doi:10.1111/cpf.12203 (2014)

33. Santos-Hiss MD, Melo RC, Neves VR, Hiss FC, Verzola RM, Silva E, Borghi-Silva A, Porta A, Montano N, Catai AM: Effects of progressive exercise during phase I cardiac rehabilitation on the heart rate variability of patients with acute myocardial infarction. Disabil. Rehabil. 33, 835-842 (2011)

34. Sawkins K, Refshauge K, Kilbreath S, Raymond J: The placebo effect of ankle taping in ankle instability. Med. Sci. Sports Exerc. 39, 781-787 (2007)

35. Shi X, Stevens GH, Foresman BH, Stern SA, Raven PB: Autonomic nervous system control of the heart: endurance exercise training. Med. Sci. Sports Exerc. 27, 1406-1413 (1995)

36. Smith ML, Hudson DL, Graitzer HM, Raven PB: Exercise training bradycardia: the role of autonomic balance. Med. Sci. Sports Exerc. 21, 40-44 (1989)

37. Task force of the European Society of Cardiology and the North American Society of Pacing and Electrophysiology: Heart rate variability: Standards of measurement, physiological interpretation, and clinical use. Circulation 93, 1043-1065 (1996) 
38. Thomas DQ, Larson BM, Rahija MR, McCaw ST: Nasal strips do not affect cardiorespiratory measures during recovery from anaerobic exercise. J. Strength Cond. Res. 15, 341-343 (2001)

39. Väinämö K, Mäkikallio T, Tulppo M, Röning J: Ms-Windows software for aerobic fitness approximation: neuroaerobic. Proceedings of the 10th Scandinavian Conference on Image Analysis. http://www.ee.oulu.fi/ research/neurogroup/Publications/scia97.pdf (1997)

40. Väinämö K, Mäkikallio T, Tulppo M, Röning J: A neuro-fuzzy approach to aerobic fitness classification: a multistructure solution to the context-sensitive feature selection problem. Neural Networks Proceedings. IEEE World Congress on Computational Intelligence. The 1998 IEEE International Joint Conference on Neural Networks 1, 797-802 (1998)

41. Väinämö K, Nissilä S, Mäkikallio T, Tulppo M, Röning J: Artificial neural networks for aerobic fitness approximation. The 1996 IEEE International Joint Conference on Neural Networks 4, 1939-1944 (1996)

42. Vanderlei LC, Silva RA, Pastre CM, Azevedo FM, Godoy MF: Comparison of the Polar S810i monitor and the ECG for the analysis of heart rate variability in the time and frequency domains. Braz. J. Med. Biol. Res. 41, 854-859 (2008)

43. Vercelli S, Ferriero G, Bravini E, Sartorio F: How much is Kinesio taping a psychological crutch? Man. Ther. 18 , e11 (2013)

44. Wallén MB, Hasson D, Theorell T, Canlon B, Osika W: Possibilities and limitations of the Polar RS800 in measuring heart rate variability at rest. Eur. J. Appl. Physiol. 112, 1153-1165 (2012)

45. Weippert M, Kumar M, Kreuzfeld S, Arndt D, Rieger A, Stoll R: Comparison of three mobile devices for measuring R-R intervals and heart rate variability: Polar S810i, Suunto t6 and an ambulatory ECG system. Eur. J. Appl. Physiol. 109, 779-786 (2010) 